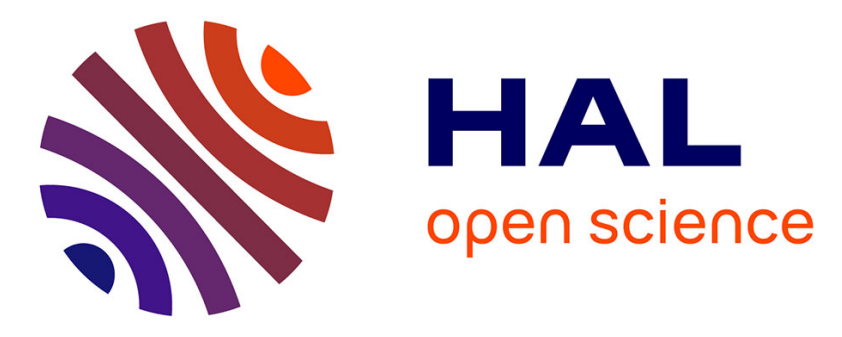

\title{
Covariance of the running range of a Brownian trajectory
}

Brandon Annesi, Enzo Marinari, Gleb Oshanin

\section{To cite this version:}

Brandon Annesi, Enzo Marinari, Gleb Oshanin. Covariance of the running range of a Brownian trajectory. Journal of Physics A: Mathematical and Theoretical, 2019, 52 (34), pp.1-14. 10.1088/17518121/ab306c . hal-02281821

\section{HAL Id: hal-02281821 \\ https: / hal.sorbonne-universite.fr/hal-02281821}

Submitted on 9 Sep 2019

HAL is a multi-disciplinary open access archive for the deposit and dissemination of scientific research documents, whether they are published or not. The documents may come from teaching and research institutions in France or abroad, or from public or private research centers.
L'archive ouverte pluridisciplinaire HAL, est destinée au dépôt et à la diffusion de documents scientifiques de niveau recherche, publiés ou non, émanant des établissements d'enseignement et de recherche français ou étrangers, des laboratoires publics ou privés. 


\section{PAPER}

\section{Covariance of the running range of a Brownian trajectory}

To cite this article: Brandon Annesi et al 2019 J. Phys. A: Math. Theor. 52345003

View the article online for updates and enhancements.

\section{IOP ebooks}

Bringing you innovative digital publishing with leading voices

to create your essential collection of books in STEM research.

Start exploring the collection - download the first chapter of every title for free. 


\title{
Covariance of the running range of a Brownian trajectory
}

\author{
Brandon Annesi ${ }^{1,2}$, Enzo Marinari ${ }^{1,2}$ and Gleb Oshanin ${ }^{3,4}$ \\ 1 Dipartimento di Fisica, Sapienza Università di Roma, P.le A. Moro 2, I-00185 \\ Rome, Italy \\ 2 INFN, Sezione di Roma 1 and Nanotech-CNR, UOS di Roma, P.le A. Moro 2, \\ I-00185 Rome, Italy \\ ${ }^{3}$ Laboratoire de Physique Théorique de la Matière Condensée (UMR CNRS 7600), \\ Sorbonne Université, CNRS, 4 Place Jussieu, 75252 Paris Cedex 05, France \\ ${ }^{4}$ Interdisciplinary Scientific Center J.-V. Poncelet (UMI CNRS 2615), Bolshoy \\ Vlasyevskiy Pereulok 11, 119002 Moscow, Russia \\ E-mail: brandonlivio@gmail.com,enzo.marinari@uniroma1.it \\ and oshanin@1ptmc.jussieu.fr
}

Received 19 February 2019, revised 19 June 2019

Accepted for publication 9 July 2019

Published 1 August 2019

\begin{abstract}
The question how the extremal values of a stochastic process achieved on different time intervals are correlated to each other has been discussed within the last few years on examples of the running maximum of a Brownian motion, of a Brownian bridge and of a Slepian process. Here, we focus on the two-time correlations of the running range of Brownian motion (BM) - the maximal extent of a Brownian trajectory on a finite time interval. We calculate exactly the covariance function of the running range and analyse its asymptotic behaviour. Our analysis reveals non-trivial correlations between the value of the largest descent (rise) of a BM from the top to a bottom on some time interval, and the value of this property on a larger time interval.
\end{abstract}

Keywords: extremal values of Brownian motion, running range, temporal correlations

(Some figures may appear in colour only in the online journal)

\section{Introduction}

Brownian motion (BM) is a paradigmatic stochastic process encountered in diverse areas of physics and chemistry [1,2], biology [2, 3], differential evolutionary games [4-6], computer science [7, 8], as well as in mathematical finance [9-12], where BM represents one of the main components in modelling of the dynamics of asset prices. 


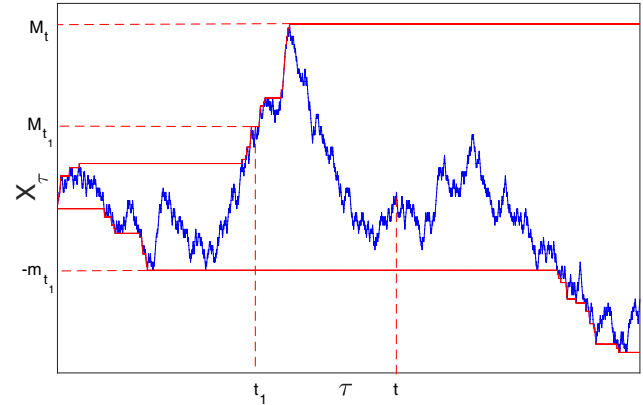

(a)

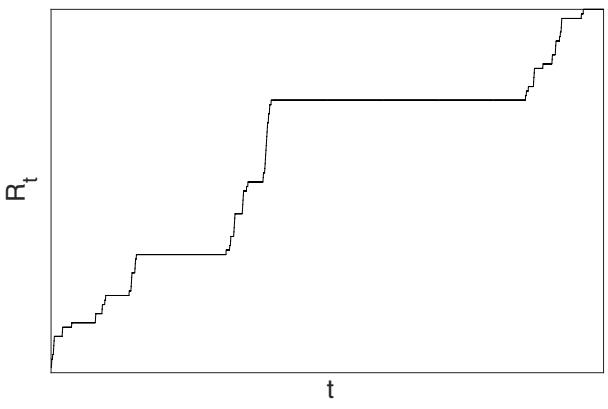

(b)

Figure 1. Panel (a): maximal and minimal displacements of a Brownian trajectory $X_{\tau}$ on time intervals $\left[0, t_{1}\right]$ and $[0, t]$. Panel (b): running range of a given BM trajectory $X_{\tau}$ depicted in panel (a).

Extreme values of BM, e.g. maximal or minimal displacements, its range (see figure 1), or first passage times to a given domain, are known to play an important role across many disciplines, since they often trigger a particular response of the system [13-19]. Since early seminal works [20-23], much effort has been invested into the analysis of statistical properties of such extreme values. In particular, the distributions of the BM maximal $/$ minimal displacements (including the joint multivariate distributions) [20-24] and of its range at a given moment of time [25-27] have been calculated exactly. Apart from the significance of these results in their own right, knowledge of such distributions is often very useful for a non-perturbative analysis of complicated functionals of BM, permitting construction of convergent bounds and hence, obtaining non-trivial exact results [28-33]. A variety of first-passage phenomena associated with the BM have been analysed using exact approaches [13-18], and there have also been some accurate approximations, which have permitted consideration of rather complicated geometries [19]. A remarkable result has been recently obtained for the distribution of the moment of time when the range of BM, evolving in presence or in absence of boundaries, first achieves some prescribed value [34].

On the other hand, the question how the extreme values of BM are correlated in time has received essentially less attention, although for many basic stochastic processes this issue has been usually addressed at the first place. This problem was approached first only within the past few years, and the two-time correlations of the running maximum of BM [35], of a Brownian bridge (a BM constrained to return to the starting point at a fixed time moment $t$ ) [36], and of a Slepian process (the difference of two BM positions taken at different time moments) [37], as well as of some records related to BM [11, 38, 39] have been determined. Here we focus on the two-time correlations (the covariance function) of the running range of Brownian motion (see figure 1) - a maximal extent (span) of a BM on a given time interval. Our analysis reveals non-trivial correlations between the value of the largest descent (rise) of a BM from the top to a bottom on some time interval, and the value of this property on a different (larger) time interval. Our exact results will be useful for constructing bounds on non-local functionals of BM, e.g. for the analysis of two-time correlations of the survival probability of a particle diffusing in presence of immobile traps, and other problems including the efficiency of diffusive foraging processes [40, 41], which depend explicitly on the range of corresponding BM. 
This paper is outlined as follows: in section 2 we introduce basic notations and present our main results. In section 3, we present the details of the derivations. Finally, in section 4 we conclude with a brief recapitulation of our results.

\section{Notations and the main results}

Let $X_{\tau}$ (with $X_{\tau=0}=0$ ) denote the position of a one-dimensional BM with diffusion coefficient $D$ at time moment $\tau \in[0, t]$ and let

$$
M_{t}=\max _{0 \leqslant \tau \leqslant t} X_{\tau} \geqslant 0, m_{t}=-\min _{0 \leqslant \tau \leqslant t} X_{\tau} \geqslant 0, \text { and } R_{t}=M_{t}+m_{t} \geqslant 0,
$$

denote the maximal positive displacement $M_{t}$ of $X_{\tau}$ within the interval $0 \leqslant \tau \leqslant t$, the minimum (i.e. the maximal negative displacement taken with a negative sign) $m_{t}$ of $X_{\tau}$ within this time interval, and the range $R_{t}$ of $X_{\tau}$ within this interval, respectively (see figure 1). Our goal is to calculate exactly the covariance function of the running range $R_{t}$, which is defined as $\mathbb{E}\left\{R_{t_{1}} R_{t}\right\}, 0 \leqslant t_{1} \leqslant t$, where the symbol $\mathbb{E}\{\ldots\}$ here and henceforth denotes averaging with respect to all possible realisations of $X_{\tau}$.

In virtue of equation (1), the covariance function of the running range can be written down as

$$
\begin{aligned}
\mathbb{E}\left\{R_{t_{1}} R_{t}\right\} & =\mathbb{E}\left\{\left(M_{t_{1}}+m_{t_{1}}\right)\left(M_{t}+m_{t}\right)\right\} \\
& =\mathbb{E}\left\{M_{t_{1}} M_{t}\right\}+\mathbb{E}\left\{m_{t_{1}} m_{t}\right\}+\mathbb{E}\left\{M_{t_{1}} m_{t}\right\}+\mathbb{E}\left\{m_{t_{1}} M_{t}\right\} .
\end{aligned}
$$

By symmetry, we have that

$$
\mathbb{E}\left\{M_{t_{1}} M_{t}\right\}=\mathbb{E}\left\{m_{t_{1}} m_{t}\right\}, \mathbb{E}\left\{M_{t_{1}} m_{t}\right\}=\mathbb{E}\left\{m_{t_{1}} M_{t}\right\},
$$

such that, eventually,

$$
\mathbb{E}\left\{R_{t_{1}} R_{t}\right\}=2\left(\mathbb{E}\left\{M_{t_{1}} M_{t}\right\}+\mathbb{E}\left\{m_{t_{1}} M_{t}\right\}\right)
$$

The first term in the brackets in the latter equation is known [35]. In contrast, the expected value of the product of a minimum and a maximum of a BM attained at two different time intervals has not been determined as yet. Our first main result is the following exact representation

$$
\begin{aligned}
\frac{\mathbb{E}\left\{m_{t_{1}} M_{t}\right\}}{D t}= & \frac{4}{\pi} \sqrt{z(1-z)}-1-z+\frac{2}{\pi} \frac{z^{3 / 2}}{\sqrt{1-z}} g_{1}\left(\sqrt{\frac{z}{1-z}}\right) \\
& +\frac{1}{\pi}\left[\left(\sqrt{\frac{z}{1-z}}+i\right) g_{1}\left(\sqrt{\frac{z}{1-z}}+i\right)+\left(\sqrt{\frac{z}{1-z}}-i\right) g_{1}\left(\sqrt{\frac{z}{1-z}}-i\right)\right]
\end{aligned}
$$

where $i=\sqrt{-1}, z=t_{1} / t \leqslant 1$, and the function $g_{1}(\phi)$ is defined by

$$
g_{1}(\phi)=\frac{1}{\phi^{2}} \int_{0}^{1} \frac{\mathrm{d} \beta}{\beta^{2}}\left(1-\frac{\pi \phi \beta}{\sinh (\pi \phi \beta)}\right) .
$$

Large- and small- $\phi$ asymptotic behaviour of $g_{1}(\phi)$ is discussed below (see equations (48) and (53)).

Recalling next the exact expression for $\mathbb{E}\left\{M_{t_{1}} M_{t}\right\}$ derived in [35], we write down the second main result of our work, which represents the desired covariance function of the running range of $\mathrm{BM}$ : 


$$
\begin{aligned}
& \frac{\mathbb{E}\left\{R_{t_{1}} R_{t}\right\}}{2 D t}=\frac{6}{\pi} \sqrt{z(1-z)}-1+\frac{2}{\pi} \arcsin (\sqrt{z})+\frac{2}{\pi} \frac{z^{3 / 2}}{\sqrt{1-z}} g_{1}\left(\sqrt{\frac{z}{1-z}}\right) \\
& +\frac{1}{\pi}\left[\left(\sqrt{\frac{z}{1-z}}+i\right) g_{1}\left(\sqrt{\frac{z}{1-z}}+i\right)+\left(\sqrt{\frac{z}{1-z}}-i\right) g_{1}\left(\sqrt{\frac{z}{1-z}}-i\right)\right] .
\end{aligned}
$$

It is instructive to consider the asymptotic behaviour of the expression in equation (7) in the limits when $t_{1} \ll t(z \rightarrow 0)$ and when $t_{1}$ is close to $t$, such that $z \rightarrow 1$. In the former limit (see the expansions in equations (51) and (52) below) we obtain

$$
\frac{\mathbb{E}\left\{R_{t_{1}} R_{t}\right\}}{\mathbb{E}\left\{R_{t_{1}}\right\} \mathbb{E}\left\{R_{t}\right\}}=1+\frac{\left(\pi^{2}-6\right)}{36} z-\frac{\left(270+7 \pi^{4}-90 \pi^{2}\right)}{10800} z^{2}+O\left(z^{3}\right),
$$

where $\mathbb{E}\left\{R_{t}\right\}=2 \mathbb{E}\left\{M_{t}\right\}=4 \sqrt{D t / \pi}$ [25-27]. Equation (8) demonstrates that correlations between $R_{t_{1}}$ and $R_{t}$ vanish when $t_{1} / t \rightarrow 0$ (which happens, namely, when $t_{1}$ is fixed and $t \rightarrow \infty)$, but this limit is approached via a slow power law, which reveals long-ranged temporal correlations between the values of the running range achieved on two different time intervals.

Within the opposite limit $z \rightarrow 1$, we find, by taking advantage of the asymptotic expansion in equation (53),

$$
\frac{\mathbb{E}\left\{R_{t_{1}} R_{t}\right\}}{\mathbb{E}\left\{R_{t}^{2}\right\}}=1-\frac{(1-z)}{2}-\frac{(1-z)^{3 / 2}}{3 \pi \ln (2)}+O\left((1-z)^{5 / 2}\right),
$$

where $\mathbb{E}\left\{R_{t}^{2}\right\}=8 \ln (2) D t[25-27]$.

The asymptotic forms in equations (8) and (9) are presented in figure 2 together with the exact result in equation (7) (solid blue curve). We observe that the small- $z$ asymptotic form in equation (8) provides a very accurate estimate of the covariance function of the running range over the whole domain of variation of $z$, and only slightly overestimates the actual value of $\mathbb{E}\left\{R_{t_{1}} R_{t}\right\}$ in the vicinity of $z=1$. In turn, the asymptotic form in equation (9), which describes the behaviour of $\mathbb{E}\left\{R_{t_{1}} R_{t}\right\}$ in the vicinity of $z=1$, is almost indistinguishable from $\mathbb{E}\left\{R_{t_{1}} R_{t}\right\}$ for $z$ as low as 0.5 .

Details of the derivation of the results in equations (5)-(9) are presented in the next section.

\section{Details of calculations}

We start with the expression for the probability $P(m \mid M)$ that a BM, commencing at the origin at $t=0$, has reached on the time interval $\left[0, t_{1}\right]$ a minimum whose absolute value $=m>0$ and on the entire interval $[0, t]$ (with $t_{1} \leqslant t$ ) a maximum $=M>0$ :

$$
\begin{aligned}
P(m \mid M)= & \int_{-m}^{M} \mathrm{~d} X \Pi_{t_{1}}(M|m| X) S_{t-t_{1}}(M-X) \\
& +\int_{-m}^{M} \mathrm{~d} X S_{t_{1}}^{*}(M|m| X) \Pi_{t-t_{1}}(M-X),
\end{aligned}
$$

where

- $S_{t}^{*}(M|m| X)$ is the conditional 'survival' probability, i.e. the probability that a BM, starting at the origin at $t=0$, attained within the time interval $[0, t]$ a minimum whose absolute value $=m$, stayed below a level $M>0$ within this interval, and at time moment $t$ appeared at position $X$ (such that $-m<X<M$ ); 


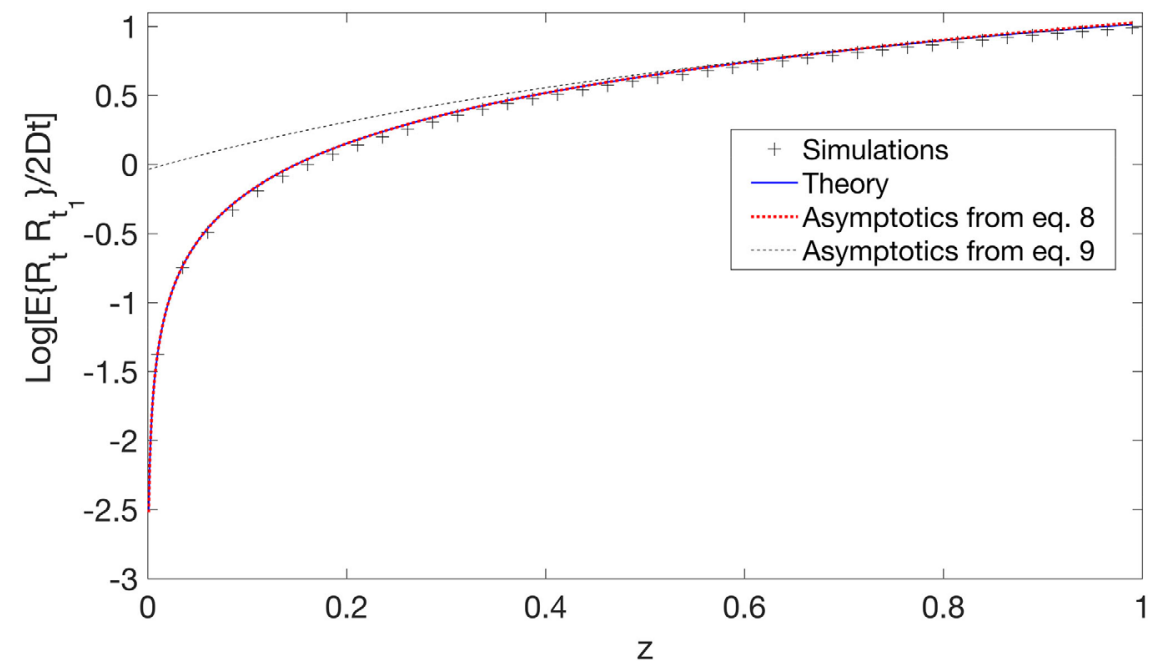

Figure 2. Exact expression for the covariance of the running range of BM in equation (7) (solid blue curve), together with the asymptotic forms in equations (8) (red dotted curve) and (9) (dotted curve). The crosses are the results of numerical simulations.

- $\Pi_{t}(M|m| X)$ is the trivariate pdf (see [23]) of the event that a BM, starting at the origin at $t=0$, attained a maximal value $=M>0$ and a minimal value $=-m$ within the time interval $[0, t]$ and ended up at position $X(-m<X<M)$ at time moment $t$;

- $S_{t}(M)$ is the standard 'survival' probability (see, e.g. [14]), i.e. the probability that a BM starting at the origin at time moment $t=0$, did not reach (stayed below) a level $M>0$ within the time interval $[0, t]$;

- $\Pi_{t}(M)$ is the pdf (see, e.g. [23]) of the event that a BM, starting at the origin at $t=0$, attained a maximal value $=M>0$ on a time interval $[0, t]$.

Let now $S_{t}(M|m| X)$ denote the standard 'survival' probability, i.e. the probability that a BM, starting at the origin, does not leave a stripe $[-m, M]$ within time interval $[0, t]$ and its endpoint at time $t$ is $X(-m<X<M)$. Clearly, we have

$$
S_{t_{1}}^{*}(M|m| X)=\frac{\partial}{\partial m} S_{t_{1}}(M|m| X)
$$

and

$$
\Pi_{t_{1}}(M|m| X)=\frac{\partial^{2}}{\partial m \partial M} S_{t_{1}}(M|m| X) .
$$

Noticing next that

$$
\Pi_{t-t_{1}}(M-X)=\frac{\partial}{\partial M} S_{t-t_{1}}(M-X),
$$

we may rewrite equation (10) formally as

$$
\begin{aligned}
P(m \mid M) & =\int_{-m}^{M} \mathrm{~d} X \frac{\partial}{\partial M}\left[S_{t_{1}}^{*}(M|m| X) S_{t-t_{1}}(M-X)\right] \\
& =\int_{-m}^{M} \mathrm{~d} X \frac{\partial^{2}}{\partial M \partial m}\left[S_{t_{1}}(M|m| X) S_{t-t_{1}}(M-X)\right] .
\end{aligned}
$$


Further on, recalling that

$S_{t-t_{1}}(M-X) \equiv \operatorname{erf}\left(\frac{M-X}{\sqrt{4 D\left(t-t_{1}\right)}}\right)=\frac{2}{\pi} \int_{0}^{\infty} \frac{\mathrm{d} z}{z} \mathrm{e}^{-4 D\left(t-t_{1}\right) z^{2}} \sin (2 z(M-X))$,

we cast equation (14) into the form

$P(m \mid M)=\frac{2}{\pi} \int_{0}^{\infty} \frac{\mathrm{d} z}{z} \mathrm{e}^{-4 D\left(t-t_{1}\right) z^{2}} \int_{-m}^{M} \mathrm{~d} X \frac{\partial^{2}}{\partial M \partial m}\left[S_{t_{1}}(M|m| X) \sin (2 z(M-X))\right]$.

Lastly, to simplify somehow our analysis, we will focus on what follows not $P(m \mid M)$ itself, but rather the distribution of an auxiliary range $\mathcal{R}=M+m$, where $M$ denotes the maximal displacement achieved on the interval $[0, t]$, while $m$ is the absolute value of the minimal displacement achieved on the subinterval $\left[0, t_{1}\right]$. This distribution is formally defined as

$$
P(\mathcal{R})=\int_{0}^{\infty} \mathrm{d} M \int_{0}^{\infty} \mathrm{d} m \delta(M+m-\mathcal{R}) P(m \mid M) .
$$

The second moment of this distribution will provide us the desired correlation function $\mathbb{E}\left\{m_{t_{1}} M_{t}\right\}$ (see equation (4)) through the relation

$$
\mathbb{E}\left\{m_{t_{1}} M_{t}\right\}=\frac{1}{2}\left(\mathbb{E}\left\{\mathcal{R}_{t}^{2}\right\}-\mathbb{E}\left\{M_{t}^{2}\right\}-\mathbb{E}\left\{m_{t_{1}}^{2}\right\}\right)
$$

The survival probability $S_{t_{1}}(M|m| X)$ is well-known (see, e.g. [23]) and is given explicitly by

$$
\begin{aligned}
S_{t_{1}}(M|m| X)= & \frac{1}{\sqrt{4 \pi D t_{1}}} \sum_{k=-\infty}^{\infty}\left[\exp \left(-\frac{(X-2 k(M+m))^{2}}{4 D t_{1}}\right)\right. \\
& \left.-\exp \left(-\frac{(X-2 M-2 k(M+m))^{2}}{4 D t_{1}}\right)\right] .
\end{aligned}
$$

For further convenience, it is however more expedient to use not the expression in equation (19), but rather its Laplace-transform $\mathcal{L}_{\lambda, \eta}$ over the 'variable' $\eta=4 D t_{1}$ :

$$
S_{\lambda}(M|m| X)=\mathcal{L}_{\lambda, \eta}\left[S_{t_{1}}(M|m| X)\right]=\int_{0}^{\infty} \mathrm{e}^{-\lambda \eta} S_{t_{1}}(M|m| X) \mathrm{d} \eta .
$$

Performing the Laplace transform and the summation over $k$, we get

$$
\begin{aligned}
& S_{\lambda}(M|m| X)=\frac{2}{\sqrt{\lambda}} \frac{\sinh (2 \sqrt{\lambda} m) \sinh (2 \sqrt{\lambda}(M-X))}{\sinh (2 \sqrt{\lambda}(M+m))}, X \geqslant 0, \\
& S_{\lambda}(M|m| X)=\frac{2}{\sqrt{\lambda}} \frac{\sinh (2 \sqrt{\lambda} M) \sinh (2 \sqrt{\lambda}(m+X))}{\sinh (2 \sqrt{\lambda}(M+m))}, \quad X \leqslant 0 .
\end{aligned}
$$

Inserting the expressions in equation (21) into equation (17) and performing all the integrations, we get

$$
P(\mathcal{R})=\frac{4}{\pi} \int_{0}^{\infty} \mathrm{d} z \mathrm{e}^{-4 D\left(t-t_{1}\right) z^{2}} \mathcal{L}_{\lambda, \eta}^{-1}\left[Q_{1}+Q_{2}+Q_{3}\right]
$$


where

$$
\begin{aligned}
& Q_{1}=\frac{\left(1-\mathrm{e}^{-2 \sqrt{\lambda} \mathcal{R}}\right)\left(1-\mathrm{e}^{-4 \sqrt{\lambda} \mathcal{R}}\right)}{\left(1+\mathrm{e}^{-2 \sqrt{\lambda} \mathcal{R}}\right)^{3}} \frac{\cos (2 z \mathcal{R})}{\lambda+z^{2}}, \\
& Q_{2}=\frac{2 \mathrm{e}^{-2 \sqrt{\lambda} \mathcal{R}}\left(1-\mathrm{e}^{-2 \sqrt{\lambda} \mathcal{R}}\right)}{\left(1+\mathrm{e}^{-2 \sqrt{\lambda} \mathcal{R}}\right)^{3}} \frac{\sin (2 z \mathcal{R})}{\sqrt{\lambda} z}, \\
& Q_{3}=\frac{\left(1-\mathrm{e}^{-2 \sqrt{\lambda} \mathcal{R}}\right)\left(1+\mathrm{e}^{-4 \sqrt{\lambda} \mathcal{R}}\right)}{\left(1+\mathrm{e}^{-2 \sqrt{\lambda} \mathcal{R}}\right)^{3}} \frac{z \sin (2 z \mathcal{R})}{\sqrt{\lambda}\left(\lambda+z^{2}\right)},
\end{aligned}
$$

and $\mathcal{L}_{\lambda, \eta}^{-1}[\ldots]$ denotes the operator of the inverse Laplace transform with respect to $\lambda$. We note that one can rather straightforwardly obtain from equation (22) the distribution of the auxiliary range in the limit $t_{1} \rightarrow 0$ with $t$ kept fixed (or equivalently, $t \rightarrow \infty$ with $t_{1}$ kept fixed) in which $\mathcal{R} \rightarrow M_{t}$, the latter being the maximum attained by a BM trajectory within the time interval $[0, t]$. In this limit (corresponding to $\lambda \rightarrow \infty$ ), we find that in the leading in $\lambda$ order

$$
Q_{1} \sim \frac{\cos (2 z \mathcal{R})}{\lambda+z^{2}},
$$

while $Q_{2}$ and $Q_{3}$ vanish. Consequently, here the distribution in equation (22) becomes

$$
P(\mathcal{R}) \sim \frac{4}{\pi} \int_{0}^{\infty} \mathrm{d} z \mathrm{e}^{-4 D t z^{2}} \mathcal{L}_{\lambda, \eta}^{-1}\left[\frac{\cos (2 z \mathcal{R})}{\lambda+z^{2}}\right] \rightarrow \frac{1}{\sqrt{\pi D t}} \exp \left(-\frac{\mathcal{R}^{2}}{4 D t}\right),
$$

which is the celebrated result due to Lévy [23] for the distribution of the maximum of a BM trajectory within the time interval $[0, t]$. The form of the distribution in the opposite limit when $t_{1} \rightarrow t$ (and hence, the auxiliary range tends to the full range, i.e. $\mathcal{R} \rightarrow R_{t}$ ), will be verified below.

Finally, we define the moments of the auxiliary range:

$$
\mathbb{E}\left\{\mathcal{R}^{n}\right\}=\int_{0}^{\infty} \mathrm{d} \mathcal{R} \mathcal{R}^{n} P(\mathcal{R}) .
$$

Changing the integration variables $z \rightarrow \sqrt{\lambda} \xi$ and $\mathcal{R} \rightarrow \rho / \sqrt{\lambda}$, we have

$$
\begin{aligned}
\mathbb{E}\left\{\mathcal{R}^{n}\right\}= & \frac{4}{\pi} \int_{0}^{\infty} \frac{\left(1-\mathrm{e}^{-2 \rho}\right)}{\left(1+\mathrm{e}^{-2 \rho}\right)^{3}} \rho^{n} \mathrm{~d} \rho \int_{0}^{\infty} \mathrm{d} \xi\left[\left(1-\mathrm{e}^{-4 \rho}\right) \frac{\cos (2 \xi \rho)}{1+\xi^{2}}\right. \\
& \left.+2 \mathrm{e}^{-2 \rho} \frac{\sin (2 \xi \rho)}{\xi}+\left(1+\mathrm{e}^{-4 \rho}\right) \frac{\xi \sin (2 \xi \rho)}{1+\xi^{2}}\right] \mathcal{L}_{\lambda, \eta}^{-1}\left[\frac{\mathrm{e}^{-4 D\left(t-t_{1}\right) \lambda \xi^{2}}}{\lambda^{1+n / 2}}\right] .
\end{aligned}
$$

The inverse Laplace transform with respect to $\lambda$ in equation (27) can now be explicitly performed. Recalling that $\eta=4 D t_{1}$, we find, explicitly,

$$
\mathcal{L}_{\lambda, \eta}^{-1}\left[\frac{\mathrm{e}^{-4 D\left(t-t_{1}\right) \lambda \xi^{2}}}{\lambda^{1+n / 2}}\right]=\frac{\left(4 D t_{1}\right)^{n / 2}}{\Gamma(n / 2+1)}\left(1-\frac{\left(t-t_{1}\right)}{t_{1}} \xi^{2}\right)^{n / 2} \theta\left(1-\frac{\left(t-t_{1}\right)}{t_{1}} \xi^{2}\right),
$$


where $\theta(x)$ is the Heaviside theta-function such that $\theta(x)=1$ for $x \geqslant 0$ and is zero otherwise.

Therefore, the following expression for the moments of the auxiliary range holds:

$$
\begin{gathered}
\mathbb{E}\left\{\mathcal{R}^{n}\right\}=\frac{2\left(D t_{1}\right)^{n / 2}}{\pi \Gamma(n / 2+1)} \int_{0}^{\infty} \frac{\left(1-\mathrm{e}^{-\rho}\right)}{\left(1+\mathrm{e}^{-\rho}\right)^{3}} \rho^{n} \mathrm{~d} \rho \int_{0}^{\infty} \mathrm{d} \xi\left[\left(1-\mathrm{e}^{-2 \rho}\right) \frac{\cos (\xi \rho)}{1+\xi^{2}}\right. \\
\left.+2 \mathrm{e}^{-\rho} \frac{\sin (\xi \rho)}{\xi}+\left(1+\mathrm{e}^{-2 \rho}\right) \frac{\xi \sin (\xi \rho)}{1+\xi^{2}}\right]\left(1-\frac{\left(t-t_{1}\right)}{t_{1}} \xi^{2}\right)^{n / 2} \theta\left(1-\frac{\left(t-t_{1}\right)}{t_{1}} \xi^{2}\right),
\end{gathered}
$$

which can be formally rewritten, upon an appropriate change of the integration variable as

$$
\begin{aligned}
\mathbb{E}\left\{\mathcal{R}^{n}\right\}= & \frac{2\left(D t_{1}\right)^{n / 2} \phi}{\pi \Gamma(n / 2+1)} \int_{0}^{\infty} \frac{\left(1-\mathrm{e}^{-\rho}\right)}{\left(1+\mathrm{e}^{-\rho}\right)^{3}} \rho^{n} \mathrm{~d} \rho \int_{0}^{1} \mathrm{~d} x\left(1-x^{2}\right)^{n / 2} \\
& \times\left[\left(1-\mathrm{e}^{-2 \rho}\right) \frac{\cos (\phi x \rho)}{1+\phi^{2} x^{2}}+2 \mathrm{e}^{-\rho} \frac{\sin (\phi x \rho)}{\phi x}+\left(1+\mathrm{e}^{-2 \rho}\right) \frac{\phi x \sin (\phi x \rho)}{1+\phi^{2} x^{2}}\right] \\
= & \frac{4\left(D t_{1}\right)^{n / 2} \phi}{\Gamma(n / 2+1)} \int_{0}^{\infty} \frac{\left(1-\mathrm{e}^{-\rho}\right)}{\left(1+\mathrm{e}^{-\rho}\right)^{3}} \exp (-\rho) \rho^{n} \mathrm{~d} \rho J_{n}(\rho),
\end{aligned}
$$

where $\phi=\sqrt{t_{1} /\left(t-t_{1}\right)}=\sqrt{z /(1-z)}$ (with $\left.z=t_{1} / t\right)$ and $J_{n}(\rho)$ is the following integral:

$J_{n}(\rho)=\frac{1}{\pi} \int_{0}^{1} \mathrm{~d} x\left(1-x^{2}\right)^{n / 2}\left[\sinh (\rho) \frac{\cos (\phi x \rho)}{1+\phi^{2} x^{2}}+\frac{\sin (\phi x \rho)}{\phi x}+\cosh (\rho) \frac{\phi x \sin (\phi x \rho)}{1+\phi^{2} x^{2}}\right]$.

It is expedient next to check the expression in equation (29) in the particular limit when $t_{1} \rightarrow t$, i.e. when the auxiliary range $\mathcal{R}$ becomes the full range of BM on the interval $[0, t]$, $\mathcal{R} \equiv R_{t}$. In this limit $\phi \rightarrow \infty$, the integral in equation (31) becomes

$$
J_{n}(\rho) \sim \frac{1}{2 \phi}
$$

in the leading in $\phi$ order, and hence we recover from equation (29) the standard expression for the moments of the range of a Brownian motion up to time $t$ [25-27]:

$$
\begin{aligned}
\mathbb{E}\left\{R_{t}^{n}\right\} & =\frac{4(D t)^{n / 2}}{\Gamma(1+n / 2)} \int_{0}^{\infty} \frac{\left(1-\mathrm{e}^{-\rho}\right)}{\left(1+\mathrm{e}^{-\rho}\right)^{3}} \exp (-\rho) \rho^{n} \mathrm{~d} \rho \\
& =\frac{4\left(2^{n}-4\right) n ! \zeta(n-1)}{2^{n} \Gamma(n / 2+1)}(D t)^{n / 2}
\end{aligned}
$$

where $\zeta(n-1)$ is the Riemann zeta-function. Note that for $n=2$ this expression has to be understood as a limit $n \rightarrow 2$. Consequently, the integral over $\rho$ entering equation (29) can be written down as

$$
\int_{0}^{\infty} \frac{\left(1-\mathrm{e}^{-\rho}\right)}{\left(1+\mathrm{e}^{-\rho}\right)^{3}} \exp (-\rho) \rho^{n} \mathrm{~d} \rho=\frac{\Gamma(n / 2+1)}{4(D t)^{n / 2}} \mathbb{E}\left\{R_{t}^{n}\right\}=\frac{\left(2^{n}-4\right) n ! \zeta(n-1)}{2^{n}},
$$

which will permit us to express the moments of the auxiliary range in terms of the moments of the full range.

To this end, we first notice that the expressions in the integrand in equation (31) admit the following expansion in the Taylor series in powers of $\rho$ : 


$$
\begin{aligned}
& \sinh (\rho) \frac{\cos (\phi x \rho)}{1+\phi^{2} x^{2}}+\cosh (\rho) \frac{\phi x \sin (\phi x \rho)}{1+\phi^{2} x^{2}}=\sum_{k=0}^{\infty} \frac{\rho^{2 k+1}}{(2 k+1) !} \sum_{m=0}^{k}(-1)^{m}\left(\begin{array}{c}
2 k \\
2 m
\end{array}\right)(x \phi)^{2 m} \\
& \frac{\sin (\phi x \rho)}{\phi x}=\sum_{k=0}^{\infty} \frac{(-1)^{k} \rho^{2 k+1}(x \phi)^{2 k}}{(2 k+1) !}
\end{aligned}
$$

Inserting these expansions into equation (31) and integrating over $x$, we arrive at the following expression for $J_{n}(\rho)$ :

$$
\begin{aligned}
J_{n}(\rho)= & \frac{\Gamma(n / 2+1)}{2 \pi}\left[\sum_{k=0}^{\infty} \frac{(-1)^{k} \Gamma(k+1 / 2) \rho^{2 k+1} \phi^{2 k}}{(2 k+1) ! \Gamma(k+n / 2+3 / 2)}\right. \\
& \left.+\sum_{k=0}^{\infty} \frac{\rho^{2 k+1}}{(2 k+1) !} \sum_{m=0}^{k} \frac{(-1)^{m} \Gamma(m+1 / 2)}{\Gamma(m+n / 2+3 / 2)}\left(\begin{array}{c}
2 k \\
2 m
\end{array}\right) \phi^{2 m}\right] .
\end{aligned}
$$

Further on, plugging equation (36) into equation (30), and integrating over $\rho$ we have

$$
\begin{aligned}
\mathbb{E}\left\{\mathcal{R}^{n}\right\}= & \frac{\left(t_{1} / t\right)^{n / 2} \phi}{2 \pi(D t)^{3 / 2}}\left[\sum_{k=0}^{\infty} \frac{(-1)^{k} \Gamma(k+1 / 2)}{(2 k+1) !} \frac{\mathbb{E}\left\{R_{t}^{2 k+n+1}\right\}}{(D t)^{k}} \phi^{2 k}\right. \\
& \left.+\sum_{k=0}^{\infty} \frac{\Gamma(k+(n+3) / 2) \mathbb{E}\left\{R_{t}^{2 k+n+1}\right\}}{(2 k+1) !(D t)^{k}} \sum_{m=0}^{k} \frac{(-1)^{m} \Gamma(m+1 / 2)}{\Gamma(m+n / 2+3 / 2)}\left(\begin{array}{c}
2 k \\
2 m
\end{array}\right) \phi^{2 m}\right],
\end{aligned}
$$

or, explicitly,

$$
\begin{aligned}
\mathbb{E}\left\{\mathcal{R}^{n}\right\}= & \frac{2\left(D t_{1}\right)^{n / 2} \phi}{\pi}\left[\sum_{k=0}^{\infty} \frac{(-1)^{k}\left(2^{2 k+n}-2\right)(2 k+n+1) ! \Gamma(k+1 / 2)}{2^{2 k+n}(2 k+1) ! \Gamma(k+(n+3) / 2)} \zeta(2 k+n) \phi^{2 k}\right. \\
& \left.+\sum_{k=0}^{\infty} \frac{\left(2^{2 k+n}-2\right)(2 k+n+1) !}{2^{2 k+n}(2 k+1) !} \zeta(2 k+n) \sum_{m=0}^{k} \frac{(-1)^{m} \Gamma(m+1 / 2)}{\Gamma(m+n / 2+3 / 2)}\left(\begin{array}{c}
2 k \\
2 m
\end{array}\right) \phi^{2 m}\right] .
\end{aligned}
$$

We concentrate next on the moments of the zeroth and of the second order, i.e. $n=0$ and $n=2$. For $n=0$ we have

$$
\begin{aligned}
\mathbb{E}\left\{\mathcal{R}^{0}\right\}= & \frac{2 \phi}{\pi}\left[2+\sum_{k=1}^{\infty} \frac{(-1)^{k}\left(1-2^{1-2 k}\right)}{(k+1 / 2)} \zeta(2 k)\right. \\
& \left.\times\left(\phi^{2 k}+\frac{(\phi-i)}{2 \phi}(\phi-i)^{2 k}+\frac{(\phi+i)}{2 \phi}(\phi+i)^{2 k}\right)\right] .
\end{aligned}
$$

Using the integral representation of the zeta-function,

$$
\zeta(s)=\frac{1}{\left(1-2^{1-s}\right) \Gamma(s)} \int_{0}^{\infty} \frac{x^{s-1} \mathrm{~d} x}{\mathrm{e}^{x}+1},
$$

which strictly holds for $\operatorname{Re} s>0$, we can perform both the summation in equation (39) and also the integral over $\mathrm{d} x$ to get

$$
\mathbb{E}\left\{\mathcal{R}^{0}\right\}=\frac{2 \phi}{\pi}\left[2+g_{0}(\phi)+\frac{(\phi-i)}{2 \phi} g_{0}(\phi-i)+\frac{(\phi+i)}{2 \phi} g_{0}(\phi+i)\right],
$$


where

$$
g_{0}(\phi)=\frac{\pi}{4 \phi}-1-2 \operatorname{arccoth}\left(\mathrm{e}^{\pi \phi}\right)+\frac{1}{2 \pi \phi}\left(\mathrm{Li}_{2}\left(\mathrm{e}^{-2 \pi \phi}\right)-4 \mathrm{Li}_{2}\left(\mathrm{e}^{-\pi \phi}\right)\right),
$$

with $\operatorname{arccoth}(\ldots)$ being the inverse hyperbolic cotangent and $\operatorname{Li}_{2}(\ldots)$ - the Euler's dilogarithm. One can readily verify that $\mathbb{E}\left\{\mathcal{R}^{0}\right\} \equiv 1$, as it should.

Next, for $n=2$ we have

$$
\begin{aligned}
\mathbb{E}\left\{\mathcal{R}^{2}\right\}= & \frac{2 D t_{1} \phi}{\pi}\left[4 \sum_{k=0}^{\infty} \frac{(-1)^{k}\left(1-2^{-2 k-1}\right)(k+1)}{(k+1 / 2)} \zeta(2 k+2) \phi^{2 k}\right. \\
& +\frac{1}{\phi^{3}} \sum_{k=0}^{\infty} \frac{(-1)^{k}\left(1-2^{-2 k-1}\right)}{(k+1 / 2)} \zeta(2 k+2)\left((2(k+1) \phi-i)(\phi+i)^{2 k+2}\right. \\
& \left.\left.+(2(k+1) \phi+i)(\phi-i)^{2 k+2}\right)\right]
\end{aligned}
$$

or, equivalently,

$$
\begin{aligned}
\mathbb{E}\left\{\mathcal{R}^{2}\right\}= & \frac{2 D t_{1} \phi}{\pi}\left(4 g_{2}(\phi)+\frac{2(\phi+i)^{2}}{\phi^{2}} g_{2}(\phi+i)+\frac{2(\phi-i)^{2}}{\phi^{2}} g_{2}(\phi-i)\right. \\
& \left.+\frac{\mathrm{i}}{\phi^{3}}\left((\phi-i)^{2} g_{1}(\phi-i)-(\phi+i)^{2} g_{1}(\phi+i)\right)\right),
\end{aligned}
$$

where the function $g_{1}(\phi)$ is defined in equation (6), while

$$
g_{2}(\phi)=\frac{1}{2 \phi} \frac{\mathrm{d}}{\mathrm{d} \phi}\left(\phi^{2} g_{1}(\phi)\right)=\frac{1}{2} g_{1}(\phi)+\frac{1}{2 \phi^{2}}\left(1-\frac{\pi \phi}{\sinh (\pi \phi)}\right) .
$$

Expressing $g_{2}(\phi)$ through $g_{1}(\phi)$ in equation (44), we find eventually

$\mathbb{E}\left\{\mathcal{R}^{2}\right\}=\frac{2 D t_{1} \phi}{\pi}\left(\frac{4}{\phi^{2}}+2 g_{1}(\phi)+\frac{\left(1+\phi^{2}\right)}{\phi^{3}}\left[(\phi+i) g_{1}(\phi+i)+(\phi-i) g_{1}(\phi-i)\right]\right)$.

To access the small- $\phi$ behaviour of $\mathbb{E}\left\{\mathcal{R}^{2}\right\}$, it is necessary to work out a convergent small$\phi$ representation of $g_{1}(\phi)$. This can be found from equation (6) and reads

$$
g_{1}(\phi)=-\frac{2}{\phi} \sum_{k=1}^{\infty} \frac{(-1)^{k}}{k} \operatorname{arctg}\left(\frac{\phi}{k}\right) .
$$

The latter representation in form of the expansion in the inverse tangents permits us to establish the following small- $\phi$ behaviour:

$$
g_{1}(\phi)=\frac{\pi^{2}}{6}-\frac{7 \pi^{4}}{1080} \phi^{2}+\frac{31 \pi^{6}}{75600} \phi^{4}+O\left(\phi^{6}\right),
$$

such that

$$
\begin{gathered}
(\phi-i) g_{1}(\phi-i)+(\phi+i) g_{1}(\phi+i)=\pi+\left(\frac{4}{3}-\frac{\pi^{2}}{9}\right) \phi^{3} \\
+\frac{\left(7 \pi^{4}+60 \pi^{2}-1440\right)}{900} \phi^{5}+O\left(\phi^{7}\right) .
\end{gathered}
$$


Consequently, we find that for small values of the parameter $\phi$ (which corresponds to either small $t_{1}$ at a fixed $t$ or large $t$ at a fixed $\left.t_{1}\right) \mathbb{E}\left\{\mathcal{R}^{2}\right\}$ obeys

$$
\mathbb{E}\left\{\mathcal{R}^{2}\right\}=2 D\left(t-t_{1}\right)\left[1+\frac{4}{\pi} \phi+\phi^{2}+\left(\frac{4}{3 \pi}+\frac{\pi}{18}\right) \phi^{3}+O\left(\phi^{4}\right)\right] .
$$

Note that for $t_{1}=0$ the leading term in the latter equation, i.e. $2 D t$, is precisely the second moment of the running maximum of a Brownian trajectory [23].

In a similar way, we construct a small-z expansion for $\mathbb{E}\left\{\mathcal{R}^{2}\right\}$. For $g_{1}(\phi)$ we get, for $z \rightarrow 0$

$$
g_{1}(\phi)=\frac{\pi^{2}}{6}-\frac{7 \pi^{4}}{1080} z+\left(\frac{31 \pi^{6}}{75600}-\frac{7 \pi^{4}}{1080}\right) z^{2}+O\left(z^{3}\right)
$$

and

$$
\begin{aligned}
& (\phi-i) g_{1}(\phi-i)+(\phi+i) g_{1}(\phi+i)=2\left(\pi-\arctan \left(\frac{2}{\phi}\right)\right) \\
& -2 \sum_{k=2}^{\infty} \frac{(-1)^{k}}{k}\left[\arctan \left(\frac{\phi-i}{k}\right)+\arctan \left(\frac{\phi+i}{k}\right)\right] \\
& =\pi+\left(\frac{4}{3}-\frac{\pi^{2}}{9}\right) z^{3 / 2}+\left(\frac{21}{80}+\frac{495-360 \pi^{2}+28 \pi^{4}}{3600}\right) z^{5 / 2}+O\left(z^{7 / 2}\right) .
\end{aligned}
$$

Equations (51) and (52), together with equation (7), yield the asymptotic expansion in equation (8).

To analyse the large- $\phi$ behaviour of $\mathbb{E}\left\{\mathcal{R}^{2}\right\}$ (which corresponds to the limit $t_{1} \rightarrow t$ ), we have to proceed in a different way. To get an appropriate large- $\phi$ representation we formally represent the integral in equation (45) over the interval $[0,1]$ as the difference of the integral over the semi-infinite interval $[0, \infty)$ and the integral over $[1, \infty)$. The first integral can be performed explicitly and is equal to $\pi \ln (2) / \phi$, while the second one can be represented as an expansion in the incomplete upper gamma functions $\Gamma(0,(2 n+1) \pi \phi)$. This gives the following exact representation of the function $g_{1}(\phi)$ :

$$
g_{1}(\phi)=\frac{\pi \ln (2)}{\phi}-\frac{1}{\phi^{2}}+\frac{2 \pi}{\phi} \sum_{n=0}^{\infty} \Gamma(0,(2 n+1) \pi \phi) .
$$

Note that for large values of $\phi$ (or $n \rightarrow \infty$ ), the leading behaviour of the incomplete gamma function follows

$$
\Gamma(0,(2 n+1) \pi \phi) \sim \frac{\exp (-(2 n+1) \pi \phi)}{(2 n+1) \pi \phi},
$$

which means that the third term in the right-hand side of equation (53) is exponentially small and can be safely neglected. In this limit we obtain

$$
\mathbb{E}\left\{\mathcal{R}^{2}\right\}=8 \ln (2) D t_{1}\left[1+\frac{1}{2 \phi^{2}}+O\left(\mathrm{e}^{-\phi}\right)\right]
$$


Note that for $t_{1}=t$, i.e. for $\mathcal{R}=R_{t}$ and $\phi=\infty$, we recover from equation (55) the classical result $\mathbb{E}\left\{R_{t}^{2}\right\}=8 \ln (2) D t$ [25-27]. The expansion in equation (55) gives directly the asymptotic form in equation (9).

\section{Conclusions}

To conclude, we studied here the covariance function of the running range $R_{t}$ of a Brownian motion-one of the extreme values of this paradigmatic stochastic process, which defines the maximal extent of a given Brownian trajectory on a time interval $[0, t]$. We have calculated exactly this two-time autocorrelation function and analysed its asymptotic behaviour. Our analysis revealed a very non-trivial form of such a correlation function and also demonstrated that the temporal correlations between the values of the running range achieved on different time intervals are very strong.

As a by-product of our analysis, we have determined the moments of an auxiliary rangethe sum of the maximal displacement achieved on the entire time interval $[0, t]$ and of the partial minimum, the minimal displacement achieved on a smaller time interval $\left[0, t_{1}\right], t_{1} \leqslant t$. These complicated expressions and the corresponding probability density function of an auxiliary range will be discussed in our future work.

We note finally that our exact results will be useful for constructing bounds on non-local functionals of BM, e.g. for the analysis of two-time correlations of the survival probability of a particle diffusing in presence of immobile traps, as well as other for problems including, e.g., the efficiency of diffusive foraging processes [40, 41], which depends explicitly on the range of corresponding $\mathrm{BM}$.

\section{Acknowledgments}

The authors acknowledge helpful discussions with Grégory Schehr and Katja Lindenberg.

\section{ORCID iDs}

Gleb Oshanin (ํ) https://orcid.org/0000-0001-8467-3226

\section{References}

[1] Metzler R and Klafter J 2000 Phys. Rep. 3391

[2] Frey E and Kroy K 2005 Ann. Phys., Lpz. 1420

[3] Berg H C 1993 Random Walks in Biology (Princeton, NJ: Princeton University Press)

[4] Oshanin G, Vasilyev O, Krapivsky P L and Klafter J 2009 Proc. Natl Acad. Sci. USA 10613696

[5] Kamimura A and Ohira T 2010 New J. Phys. 12053013

[6] Janosov M, Viragh C, Vasarhelyi G and Vicsek T 2017 New J. Phys. 19053003

[7] Majumdar S N 2005 Curr. Sci. 892076 (https://www.jstor.org/stable/24111070)

[8] Mari J-F and Schott R 2001 Probabilistic and Statistical Methods in Computer Science (New York: Springer)

[9] Williams R J 2006 Introduction to the Mathematics of Finance (Providence, RI: American Mathematical Society)

[10] Chicheportiche R and Bouchaud J-P 2014 Some applications of first-passage ideas to finance FirstPassage Phenomena and their Applications ed R Metzler et al (Singapore: World Scientific) ch 18

[11] Wergen G, Bogner M and Krug J 2011 Phys. Rev. E 83051109

[12] Oshanin G and Schehr G 2012 Quant. Finance 121325 
[13] Lindenberg K and West B J 1981 J. Stat. Phys. 42201

[14] Redner S 2001 A Guide to First-Passage Processes (Cambridge: Cambridge University Press)

[15] Condamin S, Bénichou O, Tejedor V, Voituriez R and Klafter J 2007 Nature 45077

[16] Bénichou O and Voituriez R 2014 Phys. Rep. 539225

[17] See, e.g. Metzler R, Oshanin G and Redner S (ed) 2014 First-Passage Phenomena and their Applications (Singapore: World Scientific)

[18] Grebenkov D S, Metzler R and Oshanin G 2018 Chem. Commun. 196

[19] Grebenkov D S, Metzler R and Oshanin G 2018 Phys. Chem. Chem. Phys. 2016393

[20] Kolmogorov A N 1933 Giorn. Inst. Ital. Attuari 483

[21] Smirnov N V 1939 Bull. Math. Univ. Moscou 22

[22] Feller W 1948 Ann. Math. Stat. 19177

[23] Lévy P 1948 Processus Stochastiques et Mouvement Brownien (Paris: Gauthier-Villars)

[24] Schehr G and Le Doussal P 2010 J. Stat. Mech. P01009

[25] Daniels H E 1941 Math. Proc. Camb. Phil. Soc. 37244

[26] Feller W 1951 Ann. Math. Stat. 22427

[27] Weiss G H and Rubin R G 1976 J. Stat. Phys. 14333

[28] Burlatsky S F, Oshanin G, Mogutov A and Moreau M 1992 Phys. Rev. A 45 R6955

[29] Monthus C, Oshanin G, Comtet A and Burlatsky S F 1996 Phys. Rev. E 54231

[30] Yuste S B, Oshanin G, Lindenberg K, Bénichou O and Klafter J 2008 Phys. Rev. E 78021105

[31] Oshanin G, Rosso A and Schehr G 2012 Phys. Rev. Lett. 110100602

[32] Dean D S, Gupta S, Oshanin G, Rosso A and Schehr G 2014 J. Phys. A: Math. Theor. 47372001

[33] Dean D S, Iorio A, Marinari E and Oshanin G 2016 Phys. Rev. E 94032131

[34] Wiese K J 2019 Span observables-When is a foraging rabbit no longer hungry? (arXiv:1903.06036)

[35] Bénichou O, Krapivsky P L, Mejía-Monasterio C and Oshanin G 2016 Phys. Rev. Lett. 117080601

[36] Bénichou O, Krapivsky P L, Mejía-Monasterio C and Oshanin G 2016 J. Phys. A: Math. Theor. 49335002

[37] Deng P 2017 The joint distribution of running maximum of a slepian process Methodol. Comput. Appl. Probab. $201123-35$

[38] Godreche C, Majumdar S N and Schehr G 2017 J. Phys. A: Math. Theor. 50333001

[39] Majumdar S N, von Bomhard P and Krug J 2019 Phys. Rev. Lett. 122158702

[40] Chupeau M, Bénichou O and Redner S 2016 Phys. Rev. E 93032403

[41] Bénichou O, Bhat U, Krapivsky P L and Redner S 2018 Phys. Rev. E 97022110 